\title{
Acción colectiva y diseño urbano. Notas sobre las oposiciones a la provisión de bienes de uso colectivo*
}

\author{
Francisco Báez Urbina \\ Universidad Arcis, Santiago, Chile. Email: franciscobaezurbina@hotmail.com
}

\begin{abstract}
Resumen: Los estudios de acción colectiva y de cooperación en la provisión de bienes públicos hechos desde la sociología analítica son instrumentos de gran utilidad y relevancia tanto para la reflexión sociopolítica como para el diseño de políticas públicas democráticas. En ese sentido, pensar la acción colectiva -en sentido amplio como el terreno de la constitución de lo social y de la configuración de resultados sociales relevantes desde los intereses de los diversos grupos que componen lo social- es y debiera ser de gran provecho para los cientistas sociales interesados en definir y diseñar entornos sociales más horizontales y justos territorialmente. El presente artículo recoge algunas notas de estudio emanadas de una investigación realizada en algunas ciudades hispanoamericanas respecto de los temas citados.
\end{abstract}

Palabras clave: Acción colectiva, cooperación social, bienes públicos, diseño institucional, política urbana.

\section{Collective action and urban design. Notes on the oppositions to the provision of goods for collective use}

\begin{abstract}
Studies of collective action and cooperation in the provision of public goods made from a sociological analytical instrument are useful and relevant to both social and political analysis and democratic public policy making. In that sense, thinking on collective action in a broad sense -as the grounds for the constitution of "the social" and the making of relevant social results Cumming from the interests of various social groups that form "the social"- is and should be of great use to social scientists interested in defining and designing social environments more horizontal and territorially fair. This article recovers some study notes from an investigation that was carried on in some Spanish-American cities, on the aforementioned topics.
\end{abstract}

Key words: Collective action, social cooperation, public goods, institutional design, urban policy.

\section{A ação coletiva e urbanismo. Notas sobre a oposição ao fornecimento de bens de uso coletivo}

Resumo: Estudos da ação coletiva e cooperação na provisão de bens públicos feitos a partir de uma análise sociológica são instrumentos úteis e relevantes tanto para a reflexão social e política como para a formulação de políticas públicas democráticas. Nesse sentido, pensar em uma ação coletiva em sentido 
amplo como a terra da constituição das configurações sociais e sociais relevantes resultados dos interesses dos diversos grupos sociais que compõem o que é e deve ser de grande utilidade para os cientistas sociais interessados na definição e concepção de ambientes sociais mais horizontais e territorialmente justo. Este artigo presenta alguns notas de pesquisa producto da uma investigação sobre algumas cidades hispano-americana sobre os temas mencionados.

Palavras-chave: ação coletiva, cooperação social, bens públicos, desenho institucional, política urbana.

$$
* * *
$$

\section{Introducción}

Uno de los presupuestos básicos del ajuste neoliberal de las últimas tres décadas al capitalismo reformado (Welfare State europeo o Estados Nacional Populares o modelo de Industrialización Sustitutiva de Importaciones en A. L.) involucra la promoción y legitimación del imperativo técnico por sobre consideraciones de tipo político en la constitución de la política pública. En ese tenor, suponer que el instrumental técnico esté en condiciones de proporcionar la única, mejor y, por tanto, indiscutida vía posible para la producción de bienes públicos (o bienes privados suministrados por el Estado), es por menos, interesado e ingenuo. Las políticas públicas (preferentemente las económicas aunque también las sociales) favorecen a unos intereses por sobre otros y, en la mayoría de los casos, los costes de las soluciones de política dadas se reparten inequitativamente entre los distintos grupos o estratos de la población (a nivel nacional, regional, etc.). En ese sentido, si se parte de la base de que la clase política se queja de los movimientos sociales urbanos que actualmente se levantan contra de sus decisiones, acusándolos de insolidaridad y de egoísmo por no querer un bien de uso colectivo de utilidad general (cualquier equipamiento urbano universalmente útil pero localmente no deseable), es preciso señalar: a) que ello demuestra que viven aislados del mundo de sus representados (la soberanía popular), b) que la ciudadanía reacciona movilizándose cuando desconfía de sus decisiones, y c) que ello se agrava cuando parecen ser decisiones inducidas por lobbies económicos asociados a especulativos y mezquinos intereses de clase (expresados territorialmente). Respecto de ello, y considerando -por ejemplo- que hay barrios urbanos, municipios y pueblos que la clase política sólo recuerda para poner en ellas lo que nadie quiere cerca de sus casas (lo que genera estigmatismo y malestar social localizado), ¿no sería mejor, por ejemplo, transparentar los procedimientos de decisiones y de asignación, haciendo mapas de equipamientos a nivel país, y viendo cuáles tocan a tal o a cual territorio? Pedir solidaridad al colectivo (comunidad de implantación) para un colectivo mayor de referencia (la ciudad) es estar dispuestos a compartir cargas y beneficios; esto es, por ejemplo, no enviar siempre los equipamientos indeseables a los barrios populares, y/o enviar siempre las instalaciones deseables a barrios más ricos, lo que claramente afectaría a la estructura global de coste - beneficio existente detrás de la conformación de lo urbano. 
En lo que sigue, entregamos algunas notas de estudio obtenidas sobre una investigación realizada en algunas ciudades hispanoamericanas sobre cooperación y bienes públicos. Intentaremos argumentar acerca de la idea de que los procesos de diseño de políticas públicas territoriales son procesos de carácter esencialmente político (y no técnico, como el neoliberalismo pretende señalar), donde (1) los actores involucrados representan intereses corporados orientados fundamentalmente al beneficio propio, y (2) donde dichos intereses pueden y deben ser considerados como insumos de procesos de decisión y acción colectiva generales, esto es, la acción colectiva entendida en su sentido amplio: la constitución propia de lo social.

\section{Los movimientos de oposición ciudadana contra la provisión de bienes públicos}

Proponemos definir tentativamente los movimientos de oposición ciudadana (MOC) como movimientos sociales (normalmente de carácter urbano) que reaccionan oponiéndose a aceptar un equipamiento que será de utilidad a la población en general. Si se tuviera que catalogárseles dentro de las categorías de la ciencia social estándar deberían ser ubicados dentro de lo que se debería dar en llamar "novísimos" movimientos sociales (normalmente se les asocia con territorios donde existe una larga experiencia en desarrollo democrático y movilización colectiva, aunque a veces no sea tan así). ¿La razón?, no son movimientos que estén plenamente anclados en las problemáticas socioeconómicas de carácter estructural (como el movimiento obrero de base industrial) o en las problemáticas identitarias de menor poder de convocatoria denominados desde los años 70 "nuevos movimientos sociales" (el movimiento pacifista, las identidades sexuales, las minorías étnicas, etc.). Más bien, se trataría de reacciones mucho mas heterodoxas, mucho más actuales, mucho menos masivas y mucho menos comprendidas que me atrevería a enmarcar dentro de la esfera de lo que se podría denominar como "lo social no político", es decir, el conjunto de socialidades que escapan a los anclajes estructurales económico - partidistas del capitalismo industrial (donde lo económico muchas veces coincide con lo político), y que, no demostrando vocación por el poder ni por levantar programáticas de carácter reivindicativo sectoriales, centran su accionar, o bien para oponerse (desde intereses puramente individuales promovidos por el liberalismo extremo de estos últimos 30 años) a pagar individualmente costes laterales de decisiones gubernamentales que beneficiaran al colectivo (la ciudad), o bien (desde una actitud altruista de carácter cívico y denunciante) para oponerse a decisiones, que según ellos, afectarán o bien a generaciones futuras o bien a un colectivo sólo configurable en el presente pero dentro de una escala mayor (el país, la región, etc.).

En otras palabras, entenderemos los MOC como parte de las diversas reacciones observables en el espacio de lo público y que reflejan las discrepancias existentes en lo social sobre las asimetrías de la estructura general de coste - beneficio que hay detrás de la provisión de bienes públi- 
cos (BP) (proceso de cooperación o de acción colectiva "general” o "en sentido amplio" -a escala nacional). ${ }^{1}$ En términos concretos, la existencia de MOC en operación reflejará el desacuerdo existente en lo social sobre los términos de la acción colectiva general (a escala nacional o internacional, dependiendo del bien de que se trate), es decir, sobre la distribución equitativa de la carga (los costes y/o las externalidades negativas) en la construcción de lo público. Así, que haya acción colectiva opositora en la ciudad significará que no hay acuerdo social (en términos sectoriales o territoriales) sobre la estructura general de costes presente en la provisión de BP de carácter urbano.

Pero revisemos algunas categorías teóricas de apoyo. Los BP son bienes caracterizados por la no rivalidad y la no exclusión. En otras palabras, bienes que pueden ser consumidos paralelamente por un número indefinido de agentes, y que una vez provistos, producen, disfrute universal del cual, por definición, no se puede excluir a nadie. Además de ello, generan externalidades en su entorno próximo difíciles de controlar desde mecanismos institucionales. El problema es que en ausencia de derechos de propiedad, este tipo de bienes, además de que comportan una alta complejidad en cuando a sus impactos y a los inter juegos de las externalidades que provocan, bajo los supuestos de la Teoría de la Elección Racional (TER), no generan incentivos para sostener sus propios procesos de provisión. En este contexto, el problema de los BP alude a una estructura perversa de preferencias que caracteriza el intercambio entre agentes, y que -individualmente- tiende a generar no cooperación, propiciando resultados ineficientes o sub óptimos. Sin la existencia de incentivos necesarios para la cooperación, se dará el comportamiento free rider. Que no haya cooperación significará entonces la inexistencia de acuerdos de partida para compartir los costes. Así, ningún barrio de la ciudad estará dispuesto a financiar parte de los costes de la implementación de bienes que serán de utilidad colectiva (bienes de utilidad para todos como vías urbanas, servicios públicos o territoriales en general), y el bien no se producirá.

"Un mercado competitivo -el arquetipo de las instituciones privadas- es en si mismo un BP. Una vez que se genera un mercado competitivo los individuos pueden entrar y salir libremente, ya sea que contribuyan o no con el costo de generarlo y mantenerlo. Ningún mercado puede existir por mucho tiempo sin instituciones subyacentes que lo mantengan. En escenarios de campo, más que existir en mundos aislados, las instituciones públicas y privadas con frecuencia están entretejidas y dependen una de la otra” (Ostrom, 2000: 43)

Por su parte, una acción colectiva es una acción emprendida por dos o más agentes que, bajo el ánimo de procurarse beneficios compartidos, logran establecer y coordinar esfuerzos comunes. Por el contrario, un problema de acción colectiva se dará cuando, debido a que los agentes racionales carecen de incentivos para procurar la consecución de un bien colectivo, actúan en beneficio propio y en detrimento de resultados sociales de- 
seables (Elster, 1991, 2006; Olson, 1992; Ostrom, 2000). Consistirá en una situación de intercambio en la que es mejor para todos que algunos cooperen en la consecución de los objetivos comunes trazados -sean estos cuales sean- a que nadie lo haga. Pero, aunque pueda ser mejor para todos que todos o algunos lo hagan a que nadie lo haga, desde la perspectiva de cada uno de los involucrados, siempre será mejor o más rentable no hacerlo, es decir, abstenerse de cooperar y que lo que haya que hacer para el bien de todos lo hagan otros. En escenarios descentralizados, dicho problema suele resolverse mediante una secuencia virtuosa de motivaciones individuales a la acción colectiva: utilitaristas, altruistas psicológicas, altruistas morales, etc. (Elster, 1996). En escenarios centralizados, sólo se resolverá mediante la acción de algún agente externo o principio de autoridad supra individual que decide por el colectivo de forma vinculante. Si la primera puede entenderse dentro de un orden ideal espontáneo, la segunda sólo podrá ser entendida desde una noción de necesidad de orden instituido que presuponga algún tipo de diseño institucional (Bowles, 2004).

Las notas entregadas a continuación provienen del estudio de dos casos puntuales. El primero de ellos refiere a las oposiciones de grupos organizados en México, DF. (los de la Delegación de Tláhuac a los planes de desarrollo urbano destinados a incorporar sector sur de la ciudad a la capital metropolitana mediante la construcción de la Línea 12 del metro y los del sector poniente de la capital opuestos a la construcción de vías de transporte que conectarán el Estado de México con el DF), y el segundo consiste en la oposición a las obras del paso del tren de alta velocidad por el centro de Barcelona y por debajo de monumentos patrimoniales emblemáticos para la ciudad como es el Templo de la Sagrada Familia.

\section{Algunas notas sobre los dilemas de acción colectiva y problemas involucrados: los espacios urbanos en disputa}

A continuación presentamos algunas notas de estudio sobre los dilemas de acción colectiva y sobre los problemas de interpretación y gestión involucrados.

\section{Los conflictos de intereses puestos en juego y la cuestión del poder}

En ausencia de derechos de propiedad, teniendo en cuenta los complejos efectos e impactos de sus externalidades (positivas o negativas), y considerando el problema de no cooperación generado, este tipo de bienes involucra la puesta en escena de una serie de conflictos entre los intereses de los diversos agentes involucrados. En las propias palabras de Olson (1992:25):

"No es preciso que un BP que interesa a un grupo de una sociedad beneficie necesariamente a la sociedad en conjunto. Así como una tarifa podría ser un BP para la industria que la buscó, la anulación 
de la tarifa podría ser un BP para quienes consumen el producto de la industria. Esto es igualmente cierto cuando el concepto de BP se aplica únicamente a los gobiernos. En el caso de un gasto militar, de una tarifa o de una restricción a la inmigración, que son un BP para un sólo país, también podrían ser un <mal público > para otro país y perjudiciales para la sociedad en conjunto” (M. Olson, 1992:25).

Y dentro de ello, el tema o la cuestión del tratamiento del poder es bastante complicada. Respecto de ello: "un conjunto de individuos puede ser lo bastante poderoso como para imponer el equilibrio que los favorece (...) sobre otras personas (...). Un equilibrio puede ser impuesto por aquellos que tienen más probabilidades de beneficiarse con él. Para hacerlo necesitan poder sobre los que prefieren otro equilibrio” (Elster, 1996: 112). En ese sentido, "el análisis político de los problemas de BP se contempla más adecuadamente no como un análisis del intercambio de bienes sino como un análisis del intercambio de autoridad entre actores” (Snidal en Colomer, 1991: 212). Así, en escenarios centralizados, es el Estado el que, por definición y bajo representación fiduciaria, decide por la ciudadanía definiendo qué bienes son los necesarios y en qué condiciones se deben implementar. Esto es particularmente importante pues tiene que vérselas con los intereses corporativos de diversos grupos de interés formales o informales repartidos por los territorios, lo que hace que la correcta elucidación del enmarañado y enredado entramado de relaciones de intereses y motivos a la acción colectiva apreciable en la provisión de BP sea difícilmente desentrañable y extremadamente complicada.

“.... diferencia de lo que ocurre en el intercambio de bienes privados, las decisiones (en la provisión de BP) forman siempre parte de un proceso de decisión colectiva, que cabe considerar que es de tipo político, por el cual se seleccionan las preferencias de algunas personas, se imponen las elecciones reveladas por las mismas como decisión de la sociedad y se producen los fines o resultados que aquellas decisiones comportan (...) o la libertad para que las empresas contaminen en perjuicio de los deseos de algunos ciudadanos de respirar aire limpio, o regulación de la contaminación en prejuicio de las ganancias de los empresarios y en beneficio de quienes dan prioridad a las condiciones de la respiración (...) hay siempre en la política un elemento de consenso y uno de coerción” (Colomer, 1991: 19)

\section{La dificultad de la interpretación: Los motivos y las razones de los opositores}

En términos genéricos y como patrones de comportamiento agregado estableceremos analíticamente dos tipos de movimientos: el NIMBY (not in my back yard) que es un conjunto de individuos que reaccionan oponiéndose (no cooperan) por razones de carácter egoísta a una inversión en infraestructuras públicas, y el NIABY (not in anyone's back yard) que conjunta una serie de individuos que por razones de carácter "altruista” 
igualmente se oponen a la implantación de dichos tipos de bienes. En el primer caso, se movilizan pues no se quieren pagar costes laterales indirectos de la provisión de un BP. ¿La retórica utilizada?: el autoritarismo, la falta de participación, el déficit democrático, etc. Es decir, pese a enarbolar muchas veces argumentos de carácter no estrictamente egoísta, la razón pura que mueve a los NIMBY puros es el perjuicio económico personal directo (la caída del precio del suelo, por ejemplo). Aunque hay interés colectivo en la superficie de los argumentos, apelando, por ejemplo, a razones de protección ambiental, el interés propio de los agentes opositores locales es claramente observable.

El segundo caso es más complejo e interesante pues apela al interés altruista o egoísta de segundo orden. En efecto, independientemente de los movimientos definidos como estrictamente egoístas como los NIMBY puros y que se oponen por definición al pago individual de costes, también podemos observar otros tipos de movimientos o componentes de movimientos (grupos coaligados con otros), como los ambientalistas, por ejemplo, quienes considerarán (como el político profesional) que es de interés propio trabajar para proveer beneficios colectivos; $y$ en ese sentido, se opondrán sin temor a la hipoteca trans- generacional de un BP, pues señalaran que normalmente están asociadas a oscuros y acotados intereses económicos de corto plazo (colusión de gobiernos con empresas privadas muchas veces de carácter transnacional). Estos, como señalamos en el cuerpo de la investigación nodal mencionada (Báez Urbina, 2009), presentan intereses no estrictamente egoístas que se encuentran apoyados en normas cívicas o en intereses de carácter más bien universal. Como veremos más adelante, y para analizar el tema de la naturaleza egoísta o no egoísta de los motivos de la no cooperación, particularmente importante y paradojal puede resultar el tema de la escala. En dicho sentido, en algunos casos, la no cooperación entendida como no participación, si es redefinida como abstención activa, podría adquirir significados diferentes y de contenidos progresistas y críticos ante tal o cual proyecto considerado como nefasto para el colectivo.

Para los movimientos tipo NIMBY (interés egoísta de primer orden), y en el primer sub caso mexicano, por ejemplo, las razones de este tipo de oposición que se mencionan son el congestionamiento, la sobreexplotación de los recursos y su consecuente futura escasez, la invasión de tierras ejidales para transformarlas en colonias, la inmigración (que traería muchos más problemas y demandas que serian difíciles de cumplir), el fin de las antiguas formas de agricultura tradicional, la contaminación (la creación de zonas industriales traerá consecuencias tanto para el acuífero como para la calidad del aire), problemas del transporte y vialidad (la creación de las vialidades no son una salida porque todas llegarían a las zonas de Periférico, Ermita y Zaragoza y no darían salida), el argumento de que el cambio de uso de suelo traerá graves consecuencias ambientales, etc. Se señala con ahínco que la autorización de nuevos asentamientos humanos generará la llegada de más personas y un medio social más inhumano (unidades habitacionales, industria y la línea del metro como incentivos). Se señala que es un proyecto contra las costumbres chinamperas y los usos y 
costumbres históricas de la gente pobladora de Tlahuac. Se considera como un proyecto con intereses generales (¿particulares?) sin tomar en cuenta el punto de vista de los pobladores originarios. De esta manera, se señala que "la ciudad" no quiere convivir con los recursos naturales, sino sólo explotarlos desconociendo instituciones ancestrales de producción económica local, por ejemplo. Con ello, se menciona la depredación de los recursos naturales, la violación de las leyes protectoras de las zonas de conservación, el desincentivo a la producción agropecuaria, la falta de cultura y de ganas de transmitirla en cuanto para la conservación de los recursos naturales. Se señala también la falta de información lo que provoca el problema del rumor.

Como ya señaláramos, aunque hay interés colectivo en la superficie de los argumentos, apelando -por ejemplo- a razones de protección ambiental, el interés propio de los agentes opositores locales es claramente observable: quieren mantener su industria local de carácter histórico y eso visiblemente choca con el desbocado desarrollo de la megaurbe. No obstante ello, el argumento también es atendible desde el punto de vista de la sostenibilidad anónima del colectivo. En el caso de las oposiciones en el sector poniente de la ciudad es más claro aún. Por ser grupos sociales "clase medieros” de carácter tradicional (no populares o de base agraria indígena como en el primer caso) se defienden como cuerpo de la supuesta ambición y del voraz apetito de los gestores de la ciudad por seguir intentando hacer de ella un bloque anónimo desarrollista sin límites que engulle a los sectores urbanística y socialmente ya consolidados por la ciudad en los 70 . Ahora, en el sentido de las motivaciones egoístas que hay detrás (centradas en el propio interés como la defensa de sus barrios y del precio del suelo de ellos), si bien éstos se encuentran en la base de las argumentaciones, también son acompañados y reforzados por discursos de organizaciones ambientales que apelan a la mala gestión del agua y de las cuencas acuíferas de la ciudad (se señala con claridad, por ejemplo, que el crecimiento exorbitante de la ciudad está destruyendo las bases de reproducción de sus propios recursos hídricos lo que es cierto. De hecho, en estos momentos la ciudad tiene serios problemas de reproducción y acceso al agua, un recurso histórico de carácter altamente simbólico de los habitantes de la Ciudad de México). Como vemos, el problema de la identificación de motivaciones es bastante complejo pues pese a que el auto interés -que marca los supuestos de la TER- sin duda se encuentra presente, también uno podría razonar en sentido contrario. Es decir, se podría señalar sin mayores problemas que, en su actuar, ellos defienden al colectivo y a las generaciones futuras (la ciudad sustentable) contra los que hipotecan su futuro (el gobierno y los empresarios). Volveremos sobre esto más adelante. En el caso catalán, por ejemplo, el MOC contra el paso del AVE por el centro de Barcelona, aparte de ser un movimiento símbolo del cuidado patrimonialista en la ciudad, incluye claramente el interés propio de los vecinos que viven en el eje por donde se construye el túnel subterráneo, pues creen (y de hecho es así) que dicha construcción redundará directamente en la caída del precio del suelo perjudicándolos directamente. Ello, no obstante otros tipos de intereses menos egocentrados que junto a ellos han ido conformando una bola no 
cooperativa que incluyendo organizaciones con intereses más universales como los que ahora revisaremos, dan la imagen de un movimiento homogéneo pero que tiene una composición interna variada que es preciso analizar. De esta manera, muchas veces en este tipo de movimientos las motivaciones se encuentran mezcladas o superpuestas lo que indica, en términos analíticos, que son coaliciones motivadas por intereses distintos (y a veces contrapuestos) que de manera conjunta logran establecer ruido masivo contra decisiones gubernamentales de carácter territorial que la opinión pública percibe como un todo.

Para los movimientos NIABY (interés “altruista” o egoísta de segundo orden), anotaremos separadamente algunos tópicos importantes.

La cooperación inter generacional. Independientemente del interés egoísta de primer orden que mueve a algunos MOC o a algunos de sus integrantes individuales (la dirigencias, por ejemplo) o corporados (coaliciones o coaliciones de coaliciones), hay algunos de ellos que se movilizan por otros tipos de motivos (genuinos o cuasi genuinos). ${ }^{2}$ Entre ellos, los no estrictamente egoístas (egoísmo de segundo orden o altruismo psicológico no moral que modera el auto interés). ${ }^{3}$ Ello se ve reflejado, por ejemplo, en el ánimo conservacionista del patrimonio ambiental, arquitectónico o identitario. En los casos mexicanos este animo conservacionista es clarísimo, en declaraciones como las que siguen: "el desarrollo no debe estar peleado con la conservación del territorio”, “el desarrollo no tiene que ser dañino forzosamente”, "la tecnología tiene que usarse el beneficio del hombre pero se debe buscar no dañarla", "la población pretende reflexionar a través de la información obtenida y del conocimiento de la importancia de los recursos naturales, por lo que deben ser conservados y respetados”, claramente vemos este tipo de argumentación no estrictamente egoísta que muchas veces es utilizado como arma de doble filo pues en ciertas o varias ocasiones también esconden el auto interés de grupos que claramente se ven afectados por planes de ampliación del radio urbano que los afectarán directamente. A este respecto, el caso catalán también es claro. El patronato de la Sagrada Familia, organización civil asociada a la derecha política y que gestiona la construcción del templo, se arroga indiscutiblemente el derecho a cumplir con la defensa del patrimonio arquitectónico y de la comunidad eclesial de la ciudad oponiéndose a que el túnel pase por debajo de la mega construcción arguyendo el “interés de todos” No obstante ello, detrás de dicha retórica se esconden intereses dirigenciales egocentrados o vecinos que simplemente reclaman porque su departamento se verá resquebrajado en sus cimientos.

La norma cívica y la universalidad de los intereses. Como vemos, independientemente de los movimientos definidos como estrictamente egoístas como los NIMBY puros (no cooperativos por definición) y que se oponen (deciden no cooperar) por definición al pago individual de costes, también podemos observar otros tipos de movimientos o componentes de movimientos (grupos coaligados con otros), como los ambientalistas, que consideran que es de interés propio trabajar para proveer beneficios 
colectivos y que se oponen (no cooperan) a la hipoteca "trans-generacional" de un BP en pos de "oscuros y acotados" intereses económicos de corto plazo (colusión de gobiernos con empresas privadas muchas veces de carácter transnacional). Estos, como señalamos en el cuerpo de la investigación nodal mencionada (Báez Urbina, 2009), presentan intereses no estrictamente egoístas que se encuentran apoyados en normas cívicas o en intereses de carácter más bien universal. Ahora bien, desde otra línea de argumentación, desde los estudios ambientales, y partiendo del supuesto de que el movimiento ambientalista utilizó originalmente el formato NIMBY contra la industria química, éste debería ser considerado como de carácter primario y embrionario y el NIABY como de carácter más afiatado y maduro; es decir, desde el punto de vista de la complejidad política y desde la perspectiva de los movimientos ciudadanos entendidos como agentes colectivos controladores de los decisores públicos, el NIABY debería ser entendido como una versión políticamente más compleja, más reflexiva y más avanzada que el NIMBY. No obstante ello, pasando por alto dicha línea de argumentación, pues no creemos que sea de vital importancia para nuestro análisis, desde el punto de vista de la racionalidad estándar y para efectos de este escrito, el NIMBY representa -para nosotros- el interés individual estricto y el NIABY el interés egoísta de segundo orden o altruismo psicológico (no moral). (Ambos tipos se orientan en función de sus intereses egocentrados pero los alcances de la acción y la argumentación serían distintos: uno es estrictamente egoísta y el otro no estrictamente egoísta). No obstante ello, este segundo tipo representaría un patrón de actitud mucho más interesante desde el punto de vista sociopolítico que el primero (Elster, 1991, 1996).

El tema de la escala. Para analizar el tema de la naturaleza egoísta o no egoísta de los motivos de la no cooperación, particularmente importante y paradojal es el tema de la escala. Como señala Elster (en Aguiar, 1991), en algunos casos, la "no cooperación" entendida como "no participación”, si es redefinida como "abstención activa” adquiere significados diferentes y de contenidos progresistas y críticos ante tal o cual proyecto considerado como nefasto para los “intereses generales". Así, si la lealtad de un grupo pequeño (un barrio) se traslada a uno más grande (la ciudad o el país), la cooperación puede adquirir nuevos o distintos significados. Efectivamente, en el caso de un movimiento de oposición medioambiental, por ejemplo, si un agente colectivo se opone a una determinada decisión gubernamental pues cree que este representa intereses sectarios al estar coludido con intereses empresariales, al oponerse (decide no cooperar), y aunque a vista de la opinión pública o de los medios represente la estrategia no cooperadora (diferentes casos NIABY donde la oposición es la de una ciudadanía activa, consciente y, de algún modo, “altruista”), concretamente estará contribuyendo a prevenir o a bloquear actividades corruptas que vayan teóricamente en contra del “interés general”. De esta manera, la oposición de ciertos colectivos a proyectos de provisión centralizada de BP que representan a veces la estrategia no cooperativa ante el "interés general” (como los casos NIMBY que, siendo guiados por intereses principalmente egocentrados y de carácter excluyente, impiden la localización de una in- 
fraestructura de uso público, por ejemplo), en un marco de intereses mayores como los intereses nacionales, los supranacionales o los inter generacionales (cooperación con las generaciones futuras), por ejemplo, pueden representar la estrategia opuesta, es decir, la cooperativa, en donde organizaciones de carácter altruista se oponen a la destrucción de recursos públicos (infraestructuras), comunes (pastizales) o gratuitos (aire). En ese sentido, desde el punto de vista de la obtención de transparencia en los procesos de toma de decisiones y de claridad en la rendición de cuentas públicas, a veces los MOC (NIABY) pueden lograr impactos positivos para el colectivo: frenar la especulación inmobiliaria, procesos de corrupción urbanística o de clientelismo político alentados por parte de iniciativas gubernamentales de dudosa reputación. En tal sentido, pueden considerarse instrumentos ciudadanos de control de decisiones gubernamentales espurias.

\section{Algunas notas desde la perspectiva del agente gubernamental}

Desde la perspectiva del agente gubernamental, las oposiciones sociales representan un freno importante a las agendas de inversión y a la necesidad de cumplimiento de las promesas de campaña. Así, en términos de impacto, y pese a que en ambas variantes la actividad opositora representa un freno ciudadano de alto impacto mediático a la agenda de inversiones urbanas, los resultados esperados por los opositores tienen dimensiones y alcances diferentes. En ese sentido, si en situación NIMBY lo que la no cooperación ciudadana pretende es la re-colocación del proyecto, en una situación NIABY lo que se busca es la paralización del mismo. Los incentivos selectivos (IS) son instrumentos de modificación de estructuras de preferencias (orientadas preferentemente -según la TER- a la maximización de utilidad egoísta de primer orden y que tienen por estrategia dominante no cooperar) mediante la compensación por bienes privados tangibles o intangibles y con los cuales resolver el problema de la no cooperación en la provisión de BP. En el plano de la política pública, y en el campo de los IS económicos, podremos contar con indemnizaciones, rebaja de impuestos territoriales, compensación mediante la inversión de bienes alternativos; en definitiva, cualquier tipo de iniciativa que entrañe externalidades positivas para la localidad de implantación. Por otro lado, también se ha de pensar en una interesante oferta de incentivos de carácter político, transfiriendo información a la ciudadanía afectada a modo de compensación ex ante. Se trataría, por ejemplo, de mecanismos de incorporación formal de la parte afectada a espacios de diálogo o espacios orientados a promocionar la circulación de información y, con ello, el aumento de la confianza como elemento clave dentro del campo de la cooperación. Para el neo institucionalismo, las instituciones procuran el bienestar colectivo prescribiendo universalmente la opción no preferida. Como con ello aun pueden generarse asimetrías (forced rider), la función del gestor público será la de promover un diseño global que contemple estrategias eficientes de re-equilibramiento que vayan en la dirección de inhibir cualquier posible respuesta colectiva opositora de aquellos sectores comparativamente perjudicados con la implementación del bien. Si el contrato es una estrate- 
gia correctora de la tensión entre racionalidad individual e irracionalidad colectiva, bajo las condicionantes que éste impone no todo el mundo saca igual provecho ni obtiene el mismo retorno en utilidad. En efecto, bajo el objeto de proveer bienes de uso colectivo, la implementación de restricciones externas a la comunidad (obligaciones estatales) o internas a ella (obligaciones provenientes de liderazgos internos) provoca, por un lado, el problema de la asimetría en la estructura de costes y beneficios entre agentes, y por otro, al consumo forzado (forced riding). La labor del político será entonces la de gestionar y poner en funcionamiento el contrato para intentar disolver la situación de DP de partida. En términos de política pública, esto implicará un diseño urbano equitativo en el que la estructura de costes (el peso de la carga) y beneficios puede resultar más igualitaria (planes de desarrollo urbano equitativos en los que todos pagan proporcionalmente y todos se benefician por igual).

\section{A modo de conclusiones}

Hemos partido del supuesto de que el estilo de política de carácter asociable a un tipo de razón de carácter legalista (razón burocrática), está cediendo y está dando paso a espacios de gobernanza de carácter más bien relacional y a estilos de coordinación más deliberativos y menos autoritarios. Dentro de ello, hemos intentado acercarnos a una caracterización de los MOC. Creemos que las redes críticas de base territorial "conservadoras” (NIMBY) o "progresistas" (NIABY) no están ancladas ni en los partidos políticos ni en los grupos de presión tradicionales de carácter económico típicos de la sociedad industrial fordista (obreros o patronales), sino que tienen un tipo de base de carácter territorial no partidista de cuño o bien egoísta o bien cívico de nuevo tipo. En este sentido, lo social no político egoísta (NIMBY) apelaría a la no interferencia de la política en la vida de los particulares (expresados territorialmente), en tanto, lo social no político de carácter cívico (NIABY) apelaría a un control más exhaustivo de las decisiones políticas siempre amenazadas por el descrédito y la sospecha (el interés egocentrado puro $\mathrm{v} / \mathrm{s}$ el interés altruista actuante contra los intereses supuestamente cortoplacistas de la inversión nacional transnacional en materia medioambiental, por ejemplo).

En tal sentido, coincidimos con el neo republicanismo cívico cuando señala que "la vida colectiva debe ser el producto de acuerdos colectivos entre iguales, y no el resultado de las preferencias de algunos" (Gargarella en Hernández, 2002: 97). De ahí que por fuera de las consideraciones de la libertad negativa del liberalismo, el republicanismo se preocupe por fortalecer el poder público para hacerlo permeable a la voluntad ciudadana, esto es, multiplicar los espacios para la reflexión colectiva y la deliberación conjunta acerca de lo que es la comunidad en general. (Es necesario generar más instancias de deliberación pública respecto de intentar responder qué es lo que se necesita y cuál debe ser la estructura de costes necesaria y equitativa para dar soporte a las necesidades de inversión requeridas para el bien general). En este sentido, el "Estado ausente" 
del liberalismo debe ser reemplazado por un Estado más activo en la promoción de espacios de socialidad más horizontales e inclusivos. La neutralidad liberal, debe dar paso al compromiso con una concepción del bien público y la custodia de la privacidad propia del libertarismo (Nozick) debe complementarse con tipos sociales más cercanos a motivaciones no puramente egocentradas como la virtud cívica, por ejemplo (neo republicanismo cívico).

Por último, quisiéramos señalar que, en conjetura de confianza, es decir, bajo el supuesto de que el gestor público no está en situación de colusión con intereses particulares específicos, creemos que mientras los MOC de carácter cívico son y representan una demanda por la deliberación pública en la conformación participativa de la agenda pública, los de carácter puramente egoísta son sólo agregación de intereses individuales. Por ello, y apelando al interés público y al interés del colectivo, mientras los NIABY deben ser escuchados, los NIMBY deben ser bloqueados mediante un contrato vinculante de entrada (diseño urbano equitativo y democrático) que subsuma intereses particulares en pos del logro de equilibrios sociales más justos e igualitarios.

En definitiva, las dificultades en la interpretación dicen relación con la multiplicidad de motivaciones a la acción (racionales o irracionales desde el punto de vista de la racionalidad estándar de la teoría económica) y con las diferentes razones esgrimidas por los actores en el campo de lo público, campo, por definición, de alta complejidad tanto en relación a su producción como a su gestión. No obstante ello, dichas dificultades de interpretación se pueden lograr transparentar (al menos en cierto grado) inyectando información al proceso de toma de decisiones sobre las necesidades de inversión y la estructura general (agregada) de costes involucrada, situación que acarreará, además, -y como externalidad positiva- tanto la incentivación de la participación social y política (la información diluye el dilema del prisionero), como la producción de una sensación ambiente de pertenencia (económica) a la comunidad / país.

Es preciso la producción de instrumentos de planificación, coordinación y negociación entre todas las partes involucradas tendiendo en vistas la utilización permanente de procedimientos más horizontales y deliberativos (instrumentos que pueden contribuir a crear una nueva cultura decisional y un nuevo tipo de proceso de decisiones en materia de infraestructuras y medio ambiente, por ejemplo) que transparenten y que vuelvan a evidenciar -después de 30 años de neoliberalismo extremo- que todo proceso de constitución social o urbano (y acá lo urbano sólo sirve de pretexto) no es más que el producto del conjunto de los intereses corporativos puestos en el juego político y económico general. El modelo autoritario no funciona en sociedades democráticas que ponen en práctica sus derechos y visibilizan sus intereses. Una ciudadanía empoderada exige i) la existencia de procesos deliberativos que ofrezcan buena información sobre las diferentes alternativas y sus costes, y ii) que se oigan todas las voces y todos los intereses involucrados (transparentación de intereses). 
Nótese con ello que consideramos que (1) la participación social (los ánimos de <sentirse parte> del o de los procesos) está íntimamente ligada a la participación económica (el ser y sentirse -desde el punto de vista territorial- parte de una unidad económica llamada Estado-Nación); y (2) que ambas -junto a la participación política- deben considerarse como indicadores potentes desde el punto de vista de la necesidad del análisis de la acción colectiva global (en sentido amplio y de carácter nacional) y de su promoción en un sentido virtuoso, horizontal y equitativo; esto es, no otra cosa que la construcción más igualitaria de la propia comunidad / país. Postulo aquí que así debe entenderse y que hacia ello nos debemos enfocar. 


\section{Notas}

* Preparado como notas de investigación a partir de Báez Urbina, 2009. Dicha investigación obtuvo el financiamiento de diversas fuentes de apoyo tanto en España como en Chile. Nos referimos a la Fundación Jaume Bofill y la agencia AGAUR de la Generalitat de Catalunya en España, y -en Chile- a uno de los programas de financiamiento predoctorales coordinados por CONICYT para la realización de estudios de postgrado en el extranjero.

${ }^{1}$ Donde lo social está necesariamente incrustado en la esfera de lo económico y al revés. Ver comentarios sobre diseño institucional y teoría de la elección racional de Pettit en Goodin, 2003.

${ }^{2}$ Esta argumentación es una simplificación hecha a propósito de lo ofrecido en esta pequeña presentación. El problema es de una complejidad mayor. Ver Báez Urbina, 2009 y 2006.

${ }^{3}$ Ello independiente de muchos otros tipos de motivos que por razones de espacio acá no se incluyen ni trabajan. Ver Báez Urbina, 2009. 


\section{Bibliografía}

Báez Urbina, F. (2009), “El problema de la acción colectiva: un análisis de los movimientos de oposición ciudadana a la provisión de bienes de uso colectivo”, Tesis para optar al grado de doctor en sociología por la UAB, Barcelona.

Bowles, S. (2004), Microeconomics, behavior, institutions and evolution, Princeton University Press.

Colomer, J. (comp.) (1991), Lecturas de teoría política positiva, Instituto de Estudios Fiscales, Ministerio de Economía y Hacienda, Madrid.

Elster, J. (1990), El cambio tecnológico. Investigaciones sobre la racionalidad y la transformación social, GEDISA, Barcelona.

Idem (1991), "Racionalidad, moralidad y acción colectiva”, en F. Aguiar, F. (comp.), Intereses individuales y acción colectiva, Fundación Pablo Iglesias, Madrid.

Idem (1996), Tuercas y tornillos, GEDISA, Barcelona.

Ídem (2006), El cemento de la sociedad, GEDISA, Barcelona.

Hernández, A. (comp.) (2002), Republicanismo contemporáneo. Igualdad, democracia deliberativa y ciudadanía, Siglo del Hombre Editores, Colombia.

Olson, M. (1992), La lógica de la acción colectiva. Bienes públicos y la teoría de grupos, LIMUSA Noriega Editores, México.

Ostrom, E. (2000), El gobierno de los bienes comunes, UNAM / CRIM / FCE, México.

Pettit, P. (2003), “El diseño institucional y la elección racional”, en Goodin, R. (comp.) (2003) Teoría del diseño institucional, GEDISA, Barcelona.

Snidal, D. (1991) “Bienes públicos, derechos de propiedad y organizaciones políticas”, en Colomer, J. (comp.) (1991), Lecturas de teoría política positiva, Instituto de Estudios Fiscales, Ministerio de Economía y Hacienda, Madrid.

Recibido: 24.01.2011

Aceptado: 03.03.2011 